Running Head: Reactivating Novel and Familiar Memories

\title{
Reconsolidation or Interference? Aging Effects and the Reactivation of Novel and Familiar
}

\section{Episodic Memories}

\author{
Mark L. Howe', Shazia Akhtar², Cassandra E. Bland ${ }^{1}$, and Maria V. Hellenthal ${ }^{1}$ \\ ${ }^{1}$ City, University of London \\ ${ }^{2}$ School of Life and Medical Sciences, University of Hertfordshire
}

\begin{abstract}
Authors' Note
Mark L. Howe, Shazia Akhtar, Cassandra E. Bland, and Maria V. Hellenthal, Department of Psychology, City, University of London. Shazia Akhtar is now at Hertfordshire University. We would like to thank Sash Kep, Mark Wright, and Simon Paice for their help in data collection. Correspondence concerning this research should be addressed to Prof. Mark L. Howe, Department of Psychology, City, University of London, Northampton Square, London EC1V OHB UK; e-mail: Mark.Howe.1@city.ac.uk.
\end{abstract}

\section{Editors' Note}

Dr. Fergus Craik served as editor for this article.

Revised manuscript submitted (December 6, 2019) for publication to: 


\begin{abstract}
We examined aging effects in reconsolidation and interference in episodic memory by reactivating memories for well-learned items in young and healthy older adults while controlling memory strength and the degree semantic processes contributed to memory. In Experiment 1, young and old adults learned pairs of real words and images to a strict criterion. After 24-hours, half of the images were reactivated and new words were paired with the images and learned to criterion. Following a 1-week delay, recognition and source monitoring were measured for both sets of pairings. Experiment 2 was a replication of Experiment 1, but using previously unknown novel words and unusual images. As predicted, older adults needed more trials to learn both the A-B and A-C pairings. Older adults required more trials to learn the new associations for reactivated than the not reactivated pairs, although there was no main effect of reactivation and no Age $\mathrm{x}$ Reactivation interaction for measures of recognition one-week later. These results are inconsistent with previous findings concerning age differences in reactivation effects in episodic memory. Instead, they suggest that once memory strength and input from semantic memory are better controlled, young and old adults perform similarly on tests of long-term recognition memory.
\end{abstract}

Keywords: Reactivation, Memory reconsolidation, Memory interference, Memory development, Episodic memory 


\section{Reconsolidation or Interference? Aging Effects and the Reactivation of Novel and Familiar Episodic Memories}

Once memories have formed, they are still malleable when reactivated. That is, according to reconsolidation theory, once a memory is retrieved (reactivated) it becomes unstable and subject to change during the restabilization (reconsolidation) process (e.g., Agren, 2014; Alberini \& Ledoux, 2013; Dudai, 2006; Nader, Schafe, \& LeDoux, 2000; Sara, 2000). Reconsolidation is considered adaptive in the ever changing world in which we live inasmuch as it permits memory updating through the pruning of irrelevant or inaccurate information from the trace, maintaining or increasing the relevance of a particular memory (Besnard, Caboche, \& Laroche, 2012; Lee, 2009; St. Jacques \& Schacter, 2013; St. Jacques, Montgomery, \& Schacter, 2015), and reducing the number of maladaptive (e.g., outdated) memories (Alberini \& Ledoux, 2013). More generally, because our adaptive memory system relies heavily on (re)constructive processes, both at encoding and retrieval, reconsolidation is but one mechanism that can lead to distortions in what is remembered (Schacter, 2012), distortions that can lead to false memories and misinformation effects (but see Rindal, DeFranco, Rich, \& Zaragoza, 2016).

Much of the evidence for reconsolidation comes from the animal conditioning and psychopharmacology literatures (e.g., Argen, 2014; Lee, 2009; Nader \& Hardt, 2009). For example, pharmacological work (e.g., using propranolol) with humans has shown that reconsolidation can be effective in reducing fear memories (e.g., Kindt \& Soeter, 2018). Indeed, memory editing using pharmacological agents is becoming more common in some areas of clinical practice, particularly when treating individuals who are experiencing PTSD symptoms (for a review, see Phelps \& Hofmann, 2019). 
Other studies have examined reconsolidation in humans using procedural memories (e.g., motor-sequence learning - see Walker, Brakefield, Hobson, \& Stickgold, 2003), declarative memories (e.g., paired-associate learning - see Coccoz, Maldonado, \& Delorenzi, 2011), and autobiographical memories (Schwabe \& Wolf, 2009; St. Jacques et al., 2015; St. Jacques \& Schacter, 2013). Finally, there are other studies that have examined reconsolidation in human episodic memory (e.g., Hupbach, Gomez, Hardt, \& Nadel, 2007; Hupbach, Hardt, Gomez, \& Nadel, 2008). Here, Hupbach et al. (2007) demonstrated the disruptive effects of reconsolidation on a previously learned sets of common objects (e.g., apple, comb, rock, straw). First, on Day 1 participants learned one set (List 1) of 20 objects that were each produced from a blue basket (studying them for a maximum of 4 trials or until they knew 17 of the 20 stimuli). On Day 2, following a period of consolidation, some participants were reminded of the previous task (being shown the blue basket but not asked to explicitly recall the items) whereas others were not reminded. All participants learned a second set of common objects (List 2). On Day 3, participants who had been reminded of the task from Day 1 had more List 2 intrusions when trying to recall List 1 than those who were not reminded. Hupbach et al. argued that the reminder reactivated the prior memory for List 1, returning it to a labile state where List 2 items could now become incorporated (via reconsolidation).

Although the research by Hupbach and colleagues is a promising first step toward demonstrating reconsolidation effects in human episodic memory, others see this work as simply another demonstration of reinstatement of item-context bindings (e.g., Sederberg, Gershman, Polyn, \& Norman, 2011). That is, because there exists a temporal context to the memory trace of an experience (e.g., list items), it can be used later to cue that memory. For example, the reminder (blue basket) used to cue List 1 items during reinstatement was 
then also associated with List 2 items. When participants were later asked to recall List 1 items, List 2 items would also be retrieved as they were associated with both the first and second list contexts, something that would inexorably lead to the intrusions found in the Hupbach et al. (2007) study.

Using a slightly different paradigm, one that is closely aligned to what we used in our experiments (paired-associate learning, use of nonsense materials), Forcato et al. (2007) found evidence consistent with reconsolidation effects in human declarative memory. Here, participants learned two different lists of nonsense syllable paired associates. When participants were required to learn the second list of nonsense syllable pairings $24 \mathrm{hrs}$ following the learning of a first list, this first list was resistant to interference from the second list. However, if a brief reminder of items on the first list was presented 5 min prior to learning the second list, then memory for the original list was impaired on a subsequent test. That is, reactivating items on the first list made these items more susceptible to interference from items on the second list (also see Forcato, Argibay, Pedreira, \& Maldanado, 2009).

Although these (and other) studies are encouraging, it is still not clear whether reconsolidation is the only explanation for memory-updating effects that have been observed during reinstatement manipulations in human memory. For example, a number of alternative explanations have been put forward by Rindal et al. (2016) and by Elsey, Van Ast, and Kindt (2018). Moreover, not all studies that have examined reinstatement effects in procedural and declarative memory in humans have found reconsolidation effects (e.g., see Hardwicke, Taqi, \& Shanks, 2016, for a series of replication failures). Before turning to the reasons why reconsolidation may appear and disappear across different studies, we turn to an examination of the potential impact of aging on reconsolidation. 


\section{Aging and Reconsolidation}

Aging is associated with episodic memory impairments in humans (Rajah, Kromas, Han, \& Pruessner, 2010) and other mammals (Burke \& Narnes, 2006). These aging changes are not simply confined to one's ability to recall information from memory. Indeed, older adults tend to rely more on meaning (semantic memory) in memory tasks, especially those involving recall tests. However, even on less demanding recognition memory tasks, older adults' ability to remember previously presented information is considerably reduced from that of younger adults (for a review, see Fraundorf, Hourihan, Peters, \& Benjamin, 2019).

Concerning reconsolidation effects and aging, to the best of our knowledge, only a couple of studies have examined the role of aging in memory reconsolidation. In one of these studies, Jones, Pest, Vargas, Glisky, and Fellous (2015) used reminder updating as a behavioral marker of reconsolidation. Here, the researchers examined the effects of aging on contextually-triggered reconsolidation of spatial memories in aged rats and episodic memories in aged humans. Their results produced little evidence for reconsolidation effects with aging, suggesting instead that interference, not memory updating, occurred in older participants.

In a second study, St. Jacques et al. (2015) used a museum tour paradigm to study the effects of human aging on reconsolidation-mediated updating processes. On Day 1 , participants did a physical tour of a museum with predetermined stops accompanied by audio information. On Day 2, participants were shown pictures of some of the stops from the tour as a means of reactivation. While showing these target stops, lure stops from an alternate route of the tour were sometimes shown, though the instructions for these were different. That is, for the target stops, participants had to rate the degree of reliving, or subjective recollection, in order to obtain an estimate of the quality of memory reactivation. 
For the lure stops from the route they did not take, participants were asked to rate the amount of detail, or visual information, displayed in the photo. On Day 3, participants were shown pictures of stops from the tour they attended as well as the alternate route, and they had to give yes/no judgments on whether they had visited them. They found that overall, older adults showed more false alarms than younger adults. Further, both groups gave more "yes" responses for targets and lures that had been shown on Day 2 than to baseline items shown only on Day 1, although young adults showed more of a benefit (larger advantage for reactivated over baseline items) than older adults. The authors concluded that these results demonstrate a reduced effect of reactivation-mediated updating in older adults that, to some degree, prevented strengthening of correct memories and distortions with false information. They suggested that the reason for this might be the greater difficultly older adults experience when binding information in memory.

However, before we can safely conclude what the source of age differences might be in this task, it should be noted that older adults may not have encoded the museum tour as well as younger adults. Indeed, weaker encoding usually leads to more recombination (i.e., misinformation) errors (e.g., Ecker et al., 2011), but St. Jacques et al. (2015) showed a decrease in these types of errors in older adults. Of course, this does not rule out the fact that these effects may be due to poorer relational encoding as a result of a decline in the ability to bind items together that happens in aging (Chalforte \& Johnson, 1996; NavehBenjamin, 2000). However, additional research is needed in which information encoding is better controlled (see discussion in Memory Strength section later in this Introduction).

\section{Methodological Issues Associated with Studying Reconsolidation}

Perhaps one reason for the failure to find consistent reconsolidation effects in declarative or episodic memory in humans, regardless of age, may be that it is not only 
episodic memory that is involved in learning. For example, in studies of autobiographical memory, events (e.g., going to a museum; see St. Jacques et al., 2015) involve the activation of semantic memory (e.g., the use of pre-existing knowledge about museums as well as the objects in the museum). Similarly, in the studies by Hupbach et al. $(2007,2008)$, the to-beremembered materials were all commonly known objects that participants were already familiar with in semantic memory. So when information is being reactivated it is not just the episodic connections (e.g., I learned that the experimenter wanted me to put these objects together to remember) that may come to consciousness, but also the semantic representations of these objects. Thus, reactivation in many of these studies involves both semantic and episodic components of memory.

Another problem in behavioral reconsolidation studies is that the strength of episodic memory at the termination of each of the two "learning" phases (Days 1 and 2) prior to the final memory test (Day 3 ) is unknown. If there is no assurance that all items are learned (e.g., items are presented just once, using a criterion of 17 out of 20 items correctly remembered), then we cannot be confident that items are being reactivated as some may never have been properly encoded into memory in the first place. Under these conditions, results of the final memory test on Day 3 are not particularly diagnostic of whether there are positive or negative consequences to reconsolidation as some items may never have been learned in the first place. Under these circumstances, mixed results can emerge such that weakly encoded items may be more susceptible to disruption than more strongly encoded items.

Before we present our solution to these problems, we delve into both of these issues (memory strength and relying on semantic memory in episodic tasks) in a little more depth. 
We then propose ways in which these problems can be avoided. Finally, we present two experiments where these recommendations are implemented.

\section{Memory Strength}

The issue of memory strength is an important one, both from the standpoint of understanding whether reactivation might result in reconsolidation or interference effects and also when trying to understand these effects developmentally. Concerning the latter point, different people and more critically people of different ages, take different amounts of time to learn the same information to the same extent. That older adults take longer than younger adults to learn almost any information has been known for some time (e.g., see Howe, 1988; Howe \& Hunter, 1985). Because of this, any experiment in which younger and older adults are administered the same number of learning trials almost invariably ensures that memory strength for that information will be greater in younger adults than older adults.

Concerning the former issue raised in this section, if level of learning or memory strength is left uncontrolled regardless of whether developmental issues are at play, trace strength may impact whether a reactivation treatment will evince reconsolidation or interference effects. For example, when traces that are initially weakly encoded become reactivated, they are more susceptible to reconsolidation effects as lower initial strength leaves them vulnerable to being recombined with new information. That is, as the original memory strength is low, there is less information in memory to "protect" the trace from the intrusion of new information into that trace, with the result that the new trace contains a blend of new and old information. On the other hand, when memory strength is high, reactivation may result in interference effects with the new information and the original trace rather than recombining with it. That is, strong memory traces that result from the 
original learning session can proactively interfere with subsequent attempts to learn new information. Thus, memory strength could be one of the determinants of the outcome of reactivation effects - high strength may protect the original trace making it less vulnerable to information recombination, leading to interference effects, whereas low strength traces are weak, making them more vulnerable to recombination with new information, leading to blending of information and reconsolidation effects.

In order to address this issue, a learning-to-criterion procedure was adopted in both experiments in this article. Specifically, drawing on the previous literature on controlling memory strength during learning generally (e.g., see Brainerd, Howe, \& Kingma, 1982) as well as developmentally (e.g., Brainerd, Howe, \& Desrochers, 1982; Howe, 1988; Howe \& Hunter, 1985), we had participants learn all information to a strict acquisition criterion of two consecutive errorless test trials. In this way, it is generally known that the information being learned was strongly and robustly represented in memory at the end of the acquisition session.

Differentiating Episodic and Semantic Effects

A second problem in reconsolidation research concerns the influence of semantic memory on episodic memory and learning. Reconsolidation effects are presumed to be purely episodic but frequently semantic memory becomes inexorably confounded with the episodic learning experience. Indeed, it is important to note that in most (if not all) behavioral reconsolidation studies with humans the materials being learned are not purely episodic. That is, all of the materials are previously known to the participants (e.g., words, pictures) or are related to semantic memory in some manner (e.g., involve autobiographical memory). In fact, all that is episodic about the task is how the information is being combined (e.g., in paired-associate learning) or where it is being learned (e.g., the 
laboratory context). What this means is that when information is being reactivated, both the original episodic and sematic components become active in memory. Thus, when episodic information fails, semantic information can serve to back up the original memory, potentially leading to spurious reconsolidation or interference effects. That is, when semantic memory can serve to back up information originally learned, then such memories may be quite strong during reactivation and lead to interference effects when learning new information. If semantic memory is not as strong and does not serve to back up episodic memory traces, then reactivation could lead to information recombination during reconsolidation when learning new information.

In order to address this issue, we changed stimuli across the two experiments. That is, like previous research, we used real words and pictures of real objects in Experiment 1. However, in Experiment 2 we used novel stimuli in the learning tasks in order to eliminate the semantic component of the episodic memory task. Here, we used previously normed unusual shapes paired with previously normed novel names.

\section{The Current Experiments}

The same basic procedure was used in both experiments presented here. In order to have better control over the nature of the stimuli across experiments, we opted to use a well-known paired-associate learning paradigm and associative recognition test. As noted earlier in the Introduction, Forcato et al. (2007) used a similar design and found robust reconsolidation effects. This design is also similar to that used by Allanson and Ecker (2017) where A-B pairs are presented and learned initially, followed the next day by presenting some of the stimulus A terms to implicitly reinstate the B responses, and then learn a new A-C pairing. In this design, A-C learning should be easier for reinstated than non-reinstated 
pairs if reconsolidation is operative, but harder to learn if (proactive) interference is operative (e.g., see Anderson \& Neely, 1996).

Specifically, younger and older adults learned picture-word paired associates to a stringent criterion of two consecutive errorless test trials. On Day 1 they learned 8 A-B pairs, on Day 2 they were presented with half of the original A terms, one at a time and asked to covertly remember the B term (the reactivation manipulation). They then learned a new A-C association to all 8 of the previous A-B pairings. ${ }^{1}$ Finally, on Day 3 , they took a recognition test that contained the $8 \mathrm{~A}-\mathrm{B}$ pairs, the $8 \mathrm{~A}-\mathrm{C}$ pairs, and 16 previously unseen pairs (16 new words paired with 16 new images). For each pair, participants were asked if they had seen them before (old/new pair memory judgements) and if so, whether they had seen them on Day 1 or Day 2 (source memory judgement).

Consistent with extant research, we predicted that younger adults would learn the $A-B$ and $A-C$ pairs in fewer trials than older adults. However, our critical predictions concerned whether there were different effects of reactivation across age groups (i.e., an Age $x$ Reactivation interaction). If older adults have more difficulty than younger ones at binding new information with old information already in episodic memory, then they may show interference effects and exhibit poorer memory on the recognition test for the reactivated $A-C$ pairs than the non-reactivated $A-C$ pairs. However, if older adults exhibit poorer encoding of the original A-B pairs than younger adults, and reactivation leads to greater trace lability (hence higher rates of reconsolidation for the new A-C pairing than the old A-B pairing), then older adults should exhibit better recognition memory for the

\footnotetext{
${ }^{1}$ We decided to manipulate reinstatement as a within-participant variable (also see Allanson \& Ecker, 2017) in order to obtain a more sensitive index of any reconsolidation or interference effects. When manipulated as a between-participants' variable, subsequent measures can suffer noise from individual differences.
} 
reactivated pairs for the new $\mathrm{A}-\mathrm{C}$ pairing than the original A-B pairing. Finally, once older adults learn both the A-B and A-C pairings to criterion, such that all items are well integrated and have high memory strength, we would expect little or no effect of reactivation. Thus, if criterion learning leads to strong memory traces, then this could lead to few or no differences in the recognition of reactivated A-C pairs, non-reactivated A-C pairs, and the original A-B pairings.

\section{Experiment 1}

In this first experiment, we tested these Age $x$ Reactivation predictions using a criterion learning procedure to circumvent general individual differences in learning rates and well as developmental differences in acquisition.

\section{Participants}

To determine the sample size, a power analysis was conducted, which showed a total of 170 participants ( 85 in each group) were needed when $d$ was estimated at 0.5 and power was estimated at .80 . There were 89 young adults ( 26 males and 63 females) and 89 older adults (30 males and 59 females), the characteristics of which are shown in Table 1. The older adults were volunteers who were community dwelling, did not present with memory complaints or suffer from depression, had normal cognitive functioning (as assessed by the Mini Mental State Examination, MMSE: Folsten, Folstein, \& McHugh, 1974) and activities of daily living. In addition, all older adults completed the National Adult Reading Tests (NART) which can be used as a proxy for IQ (Nelson, 1982). 


\section{Design and materials}

We used a two condition (Reactivation vs. No reactivation) mixed design. The stimuli consisted of 8 real images taken from the Bank of Standardized Stimuli (BOSS), (Mathieu, Dionne-Dostie, Montreuil, \& Lepage, 2010) and 16 name nouns, taken from Rubin and Friendly's (1986) word norms. The images were presented in color with the text in black on a white background (see Figure 1).

Insert Figure 1 about here

\section{Procedure}

Participants were tested individually in a laboratory at City, University of London. The study was conducted in three stages. Sessions 1 and 2 took place on consecutive days and Session 3 was approximately six to seven days later.

In Session 1, the A-B learning phase, all participants were shown eight pairings consisting of novel images (A term) with unusual names (B term). Participants were shown each pairing one at a time for 500 milliseconds each, with the name read out aloud by the researcher. After all eight pairings were presented, participants were exposed to the images (A term) one at a time and were asked to report the corresponding name for that item (B term). Regardless of whether the response was correct or incorrect, participants were immediately given feedback (the image with the correct label). This procedure (familiarly known in the paired-associate learning literature as an anticipation learning procedure) was 
repeated until all eight pairings were tested and participants correctly reported the pairings on two consecutive learning trials.

In Session 2 one day later, the A-C learning phase was presented. Participants were presented with half of the images from Session 1 ( $A$ term only) and were asked to silently remember their names ( $\mathrm{B}$ term). Participants were asked not to overtly report the associated name. Immediately after, participants entered the A-C learning phase in which they were required to learn the $A$ terms (novel images) in association with new unusual names ( $C$ term). The learning procedure for the A-C pairings took place in the exact same manner as the A-B learning phase. Which of the 4 pairs from Session 1 were reactivated at the beginning of Session 2 was counterbalanced across participants.

Session 3 was conducted six to seven days after Session 2, and consisted of a standard recognition test. Participants were shown randomly the original eight A-B pairings from Session 1 and the eight A-C pairings from Session 2 with a further 16 unseen foil pairings. Participants were required to indicate whether the pairing was old (have seen it before) or new (never encountered before). Additionally, for the old responses participants were required to report if they remembered the pairing from either the first or second session.

\section{Results and Discussion}

Session 1: A-B pairings

The number of learning trials needed to reach criterion $(2$ consecutive correct test trials) was analyzed between age groups for Session 1 acquisition of the A-B pairs. The trials were also analyzed according to whether the A terms were to be reactivated at Session 2 to ensure there were no pair learning confounds that would carry over to Session 2. A 2 (Reactivation: reactivation at Session 2 vs. no reactivation at Session 2) 22 (Age: young vs. 
old) analysis of variance (ANOVA) was conducted on the A-B learning trials. As expected, there was a significant main effect of age, $F(1,176)=47.09, p<.001, \eta_{p}^{2}=.0$, where younger adults took fewer learning trials $(M=2.81, S D=1.18)$ than older adults to reach acquisition criterion $(M=4.06, S D=1.28)$. There was no main effect of the Reactivation and no significant interaction effect of Age $\mathrm{x}$ Reactivation (all ps ns; see Table 2).

\section{Session 2: A-C pairings}

The key variable of interest was the measure of learning trials to criterion at Session 2 with the new $C$ terms. A 2 (Reactivation: reactivation vs. no reactivation) $\times 2$ (Age: young vs. old) ANOVA was conducted on the A-C learning trials. As expected, there was a significant main effect of age, $F(1,176)=75.9, p<.001, \eta_{p}^{2}=.3$, where younger adults took fewer learning trials $(M=2.84, S D=0.98)$ than older adults to reach acquisition criterion $(M$ $=4.23, S D=1.27$ ). Surprisingly, there was no main effect of Reactivation ( $p$ ns; see Figure 2 ) and no significant interaction effect of Age x Reactivation ( $p$ ns; see Table 2).

Insert Figure 2 about here

Exploratory analysis. Although the interaction was not significant, we conducted exploratory Bonferroni corrected post hoc tests within each age group. Consistent with the main analysis, there was no effect of the reactivation conditions on the younger adults' AC learning trials ( $p$ ns). However, for the older adults, significantly more AC learning trials were needed for the reactivated pairs $[t=2.5, p<.05(M=4.3, S D=1.23)$ compared to those that were not reactivated $(M=4.14, \mathrm{SD}=1.24)]$. 


\section{Session 3: Recognition test}

Separate 2(age) $\times 2$ (reactivation) $\times 2$ (pairing) repeated measures ANOVAs were conducted for the recognition responses and source monitoring accuracy rates. For recognition rates, there was no significant main effect of reactivation or age (all $p$ 's NS). There were no significant interaction effects. For source monitoring responses there were no significant results (either in terms of main effects or interactions; see Table 2).

Insert Table 2 about here

Because the critical Age $x$ Reactivation interaction did not achieve statistical significance using traditional analyses, we used Bayesian analyses in order to evaluate whether these recognition data were consistent with the null or alternative hypothesis. We calculated a Bayes factor with a default prior of 0.2 (Wagenmakers et al., 2018). We found a $\mathbf{B F}_{10}$ of 0.158 for reactivation and $\mathbf{B F}_{10}$ of 0.03 for the Age $\mathrm{x}$ Reactivation interaction. As a consequence, these data are more consistent with the null than alternative hypothesis.

\section{Experiment 2}

Experiment 1 demonstrated that reactivation did not result in memory impairment or improvement in either younger or older adults even when a strict learning criterion was used to ensure adequate strength of pair information in memory. In this second experiment, we attempt to extend these findings to conditions in which there is little (or no) additional learning support available from semantic memory. Here, instead of pairing known pictures with known words, we paired novel pictures with novel non-words. 


\section{Participants}

To determine the sample size, a power analysis was conducted, which showed a total of 170 participants ( 85 in each group) were needed when $d$ was estimated at 0.5 and power was estimated at .80. There were 89 new young adults ( 36 males and 53 females) and 86 new older adults ( 28 males and 58 females) whose characteristics are shown in Table 1. The older adults were volunteers who were community dwelling, did not present with memory complaints or suffer from depression, had normal cognitive functioning (as assessed by the Mini Mental State Examination, MMSE: Folstein, Folstein, \& McHugh, 1974) and activities of daily living. In addition, all older adults completed the National Adult Reading Tests (NART) which can be used as a proxy for IQ (Nelson, 1982).

Design and materials

Like Experiment 1, Experiment 2 used a two condition (Reactivation vs. No reactivation) mixed design. The stimuli consisted of 8 novel images and 16 unusual names (see Figure 3) taken from the Novel Object and Unusual Name (NOUN) database (Horst \& Hout, 2015). The study consisted of three sessions with all sessions running on a PC screen. For Session 1, 8 novel images and their corresponding 8 unusual names were paired (referred to as the A-B pairings) and presented. For Session 2, 4 of the previously presented novel images from Session 1 (A terms) were presented followed by re-pairing of all of the original 8 novel images presented in Session 1 with new corresponding unusual name pairings ( $A-C$ pairings). The novel images were presented in color with the text in black on a white background. 


\section{Procedure}

The procedure was identical to Experiment 1. However, where Experiment 1 paired 8 real images and real words, in Experiment 2, we paired unusual images and novel names.

\section{Results and Discussion}

Session 1: A-B pairings

The number of learning trials needed to reach criterion ( 2 consecutive correct test trials) was analyzed between age groups for Session 1 learning of the A-B pairs. The trials were also analyzed according to whether the A terms were to be reactivated at Session 2 to insure there were no pair-learning confounds that would carry over to Session 2. A repeated measures ANOVA was conducted with the learning trials for the A-B pairings. As expected, there was a significant main effect of age, $F(1,173)=57.3, p<.001, \eta_{p}^{2}=247$, where younger adults took fewer learning trials $(M=3.88, S D=1.38)$ compared to the older adults $(M=5.38, S D=1.4)$. As expected, there was no main effect of the reactivation condition at Session 1 (hence there was no initial learning bias that would carry over to Session 2), and no significant interaction (all ps ns; see Table 2).

\section{Session 2: A-C pairings}

The key variable of interest was the measure of learning trials to criterion at Session 2 with the new $C$ terms. A repeated measures ANOVA was conducted for the learning trials with reactivation as the within-participant variable and age as the between-participants' variable. Unexpectedly again, there was no main effect of reactivation ( $p$ ns; see Figure 4) and no Age $\mathrm{x}$ Reactivation interaction ( $p=\mathrm{ns})$. As expected, there was a significant main 
effect of age, $F(1,172)=57.2, p<.001, \eta_{p}^{2}=.26$. Younger adults took fewer learning trials $(M=3.9, S D=1.31)$ compared to the older adults $(M=5.32, S D=1.32)$.

Insert Figure 4 about here

Exploratory analysis. Although the interaction was not significant, we conducted exploratory Bonferroni corrected post hoc tests within each age group. Consistent with the main analysis, there was no effect of the reactivation conditions on the younger adults' AC learning trials ( $p$ ns). However, for the older adults, significantly more AC learning trials were needed for the reactivated pairs $[t=2.14, p<.05 ;(M=5.39, S D=1.32)]$ compared to those that were not reactivated $(M=5.26, S D=1.42)$.

\section{Session 3: Recognition test}

Separate 2(age) $\times 2$ (reactivation) $\times 2$ (pairing) repeated measures ANOVAs were conducted for the recognition responses and source monitoring accuracy rates. For recognition rates, there was no significant main effect of reactivation or age (all $p$ 's NS). There were no significant interaction effects. For source monitoring responses there were no significant results (either in terms of main effects or interactions). See Table 2.

As the critical Age $x$ Reactivation interaction did not achieve significance using traditional analyses, we again used Bayesian analyses in order to evaluate whether these recognition data were consistent with the null or alternative hypothesis. We calculated a Bayes factor with a default prior of 0.2 (Wagenmakers et al., 2018). We found a $\mathbf{B F}_{10}$ of 0.15 for reactivation and $\mathbf{B F}_{10}$ of 0.04 for the Age $\times$ Reactivation interaction. As a consequence, these data are more consistent with the null than alternative hypothesis. 


\section{General Discussion}

The purpose to these two experiments was to examine whether reactivation resulted in the weakening of earlier learned A-B associations with subsequent reconsolidation of new $\mathrm{A}-\mathrm{C}$ associations into the old $\mathrm{A}-\mathrm{B}$ trace, or in strengthening earlier learned A-B associations with subsequent (proactive) interference of the old A-B information with remembering the new A-C information. We also sought to examine whether these effects varied with age in adulthood. We tested these possible Age $x$ Reactivation interactions when memory strength of the original A-B pairs and subsequently learned A-C pairs was controlled using criterion learning procedures (both experiments) and when semantic memory could not serve to support episodic memory (Experiment 2).

When we examined the learning data, unsurprisingly we found that (1) younger adults took fewer trials to learn pairs than older adults (both $A-B$ and $A-C$ ) and ( 2 ) although the interaction was not significant, post-hoc analyses indicated that older adults took longer to learn reactivated than nonreactivated $\mathrm{A}-\mathrm{C}$ pairs. Concerning 1 , this is a fairly typical effect in the aging literature with myriad studies showing this learning lag (e.g., Howe, 1988; Howe \& Hunter, 1985). Concerning 2, if anything, this finding is more reminiscent of proactive interference than reconsolidation - that is, reminding older adults of the previous association made it more difficult to learn a new association. This finding is consistent with other recent research showing that older adults are more prone to proactive interference effects in AB-AC associative memory than younger adults (Burton, Lek, Dixon, \& Caplan, 2019). Indeed, because pairs in both of our experiments were learned to a strict criterion, reactivating those pairs after a delay served to further strengthen them, making the learning of new associations more difficult for those pairs than the ones older adults had not been reminded of prior to the learning of new A-C pairs. Of course, given that this finding is 
simply a trend, one not backed up by a significant interaction, further research is needed to determine the precise theoretical nature of this effect.

However, the critical test of whether there are age differences in memory due to reactivation (reconsolidation or interference) comes when we examine the final recognition test for both the original A-B pairings and the subsequently learned $A-C$ pairings. Across both experiments, not only did we fail to find a main effect for reactivated pairs, there was no Age $x$ Reactivation interaction. Bayesian analyses confirmed that the data from both of these experiments were more consistent with the null than alternative hypothesis. Thus, when episodic memory for information that has been well-learned (high memory strength) is tested with (Experiment 1) or without (Experiment 2) semantic memory backup, age differences in memory performance are hard to find. More specifically, although there are clear ceiling effects in the recognition scores, it is important to note that in both experiments when learning was equated between the two age groups there were no effects of aging on recognition performance.

\section{Caveats and Limitations}

It could be argued that because our reactivation manipulation was within rather than between participants that the reactivation of a subset of the $A B$ terms caused the remaining $A B$ pairs to also be reactivated, albeit covertly. If so, then we should see all of the AC terms being either easier (reconsolidation) or harder (strengthening/interference) to learn following reactivation. Although this was the case for younger adults, there was a trend for older adults such that for those $A B$ pairs that were overtly reactivated, it was harder to learn the $A C$ pairs than for those $A B$ pairs that were not directly reactivated. It would seem then that, at least for older adults, reactivation was effective and it was confined to those $A B$ pairs that were directly/overtly reactivated. Thus, reactivation 
manipulations, even when used as a within-participant variable, can successfully target specific pairings while at the same time leave the non-targeted pairings inactive (also see Allanson \& Ecker, 2017). Indeed, the advantage of using a within-participant reactivation manipulation is that it can reduce noise created by individual differences.

It could also be argued that by not including a parallel series of non-criterion learning conditions, we cannot be certain that criterion learning was central to the absence of age differences. Although this is a fair point, there exists considerable research in which noncriterion learning procedures have been employed, some of which produce reconsolidation effects (e.g., Forcato et al., 2007; Hupbach et al., 2007) and some of which do not (e.g., Allanson \& Ecker, 2017). Although there are any number of methodological differences among these studies that could have led to these different results, it is well known that by using criterion learning, memory differences across age in childhood (Howe, 2002) as well as adulthood (e.g., Howe, 1988; Howe \& Hunter, 1985) are reduced or eliminated. As such, criterion learning remains a valuable tool when examining age differences in long-term memory and retention, ones that are independent of initial learning differences that originate in failures to adequately acquire the information in the first place (i.e., at encoding).

\section{Conclusion}

Our results are inconsistent with the view that reconsolidation of well-learned episodic memories, whether the items memorized are familiar or completely novel, can change or alter the original traces. Rather, these results are more consistent with the view that reactivation strengthens (not weakens) the original trace, making it more difficult to learn new information, at least for older adults. This greater difficulty in learning is consistent with much of the literature on aging and episodic memory. 


\section{References}

Agren, T. (2014). Human reconsolidation: a reactivation and update. Brain Research Bulletin, 105, 70-82.

Alberini, C. M., \& LeDoux, J. E. (2013). Memory reconsolidation. Current Biology, 23, R746R750.

Allanson, F., \& Ecker, U. K. H. (2017). No evidence for a role of reconsolidation in updating paired associates. Journal of Cognitive Psychology, 29, 912-919.

Anderson, M. C., \& Neely, J. H. (1996). Interference and inhibition in memory retrieval. In E. L. Bjork \& R. A. Bjork (Eds.), Memory. Handbook of perception and cognition ( $2^{\text {nd }}$ Ed., pp. 237-313). San Diego, CA: Academic Press.

Besnard, A., Caboche, J., \& Laroche, S. (2012). Reconsolidation of memory: a decade of debate. Progress in Neurobiology, 99, 61-80.

Brainerd, C. J., Howe, M. L., \& Desrochers, A. (1982). The general theory of two-stage learning: A mathematical review with illustrations from memory development. Psychological Bulletin, 91, 634-665.

Brainerd, C. J., Howe, M. L., \& Kingma, J. (1982). An identifiable model of two-stage learning. Journal of Mathematical Psychology, 26, 263-293.

Burton, R. L., Lek, I., Dixon, R. A., \& Caplan, J. B. (2019). Associative interference in older and younger adults. Psychology and Aging, 34, 558-571.

Chalforte, B. L., \& Johnson, M. K. (1996). Feature memory and binding in young and older adults. Memory \& Cognition, 24, 403-416. 
Coccoz, V., Maldonado, H., \& Delorenzi, A. (2011). The enhancement of reconsolidation with a naturalistic mild stressor improves the expression of a declarative memory in humans. Neuroscience, $185,61-72$.

Dudai, Y. (2006). Reconsolidation: the advantage of being refocused. Current opinion in neurobiology, 16, 174-178.

Ecker, U. K. H., Lewandowsky, S., Swire, B., \& Change, D. (2011). Correcting false information in memory: Manipulating the strength of misinformation encoding and its retraction. Psychonomic Bulletin \& Review, 18, 570-578.

Elsey, J. W. B., Van Ast, V. A., Kindt, M. (2018). Human memory reconsolidation: A guiding framework and critical review of evidence. Psychological Bulletin, 144, 797-848.

Forcato, C., Argibay, P. F., Pedreira, M. E., \& Maldonado, H. (2009). Human reconsolidation does not always occur when a memory is retrieved: The relevance of the reminder structure. Neurobiology of Learning and Memory, 91, 50-57.

Forcato, C., Burgos, V. L., Argibay, P. F., Molina, V. A., Pedreira, M. E., \& Maldonado, H. (2007). Reconsolidation of declarative memory in humans. Leearning \& Memory, 14, 295-303.

Fraundorf, S. H., Hourihan, K. L., Peters, R. A., \& Benjamin, A. S. (2019). Aging and recognition memory: A meta-analysis. Psychological Bulletin, 145, 339-371.

Hardwicke, T. E., Taqi, M., \& Shanks, D. R. (2016). Postretrieval new learning does not reliably induce human memory updating via reconsolidation. Proceedings of the National Academy of Sciences USA, 113, 5206-5211.

Howe, M. L. (2011). The adaptive nature of memory and its illusions. Current Directions in Psychological Science, 20, 312-315. 
Howe, M. L. (2002). The role of intentional forgetting in reducing children's retroactive interference. Developmental Psychology, 38, 3-14.

Howe, M. L. (1988). Measuring memory development in adulthood: A model-based approach to disentangling storage-retrieval contributions. In M. L. Howe and C. J. Brainerd (Eds.), Cognitive development in adulthood (pp. 39-64). New York: SpringerVerlag.

Howe, M. L., \& Hunter, M. A. (1985). Adult age difference in storage-retrieval processes: A stages-of-learning analysis of developmental interactions in concreteness effects. Canadian Journal of Experimental Psychology, 39, 130-150.

Hupbach, A., Gomez, R., Hardt, O., \& Nadel, L. (2007). Reconsolidation of episodic memories: A subtle reminder triggers integration of new information. Learning \& Memory, 14, 47-53.

Hupbach, A., Gomez, R., \& Nadel, L. (2009). Episodic memory reconsolidation: Updating or source confusion? Memory, 17, 502-510.

Hupbach, A., Hardt, O., Gomez, R., \& Nadel, L. (2008). The dynamics of memory contextdependent updating. Learning \& Memory, 15, 574-579.

Jones, B. J., Pest, S. M., Vargas, I. M., Glisky, E. L., \& Fellous, J.-M. (2015). Contextual reminders fail to trigger memory consolidation in aged rats and aged human. Neurobiology of Learning and Memory, 120, 7-15.

Kindt, M., \& Soeter, M. (2018). Pharmacologically induced amnesia for learned fear is time and sleep dependent. Nature Communications, 9:1316, 1-10.

Lee, J. L. (2009). Reconsolidation: maintaining memory relevance. Trends in Neurosciences, 32, 413-420. 
Nadel, L., Hupbach, A., Gomez, R., \& Newman-Smith, K. (2012). Memory formation, consolidation and transformation. Neuroscience and Biobehavioral Reviews, 36, $1640-1645$.

Nader, K., \& Hardt, O. (2009). A single standard for memory: the case for reconsolidation. Nature Reviews Neuroscience, 10, 224-234.

Nader, K., Schafe, G. E., \& LeDoux, J. E. (2000). The labile nature of consolidation theory. Nature Reviews Neuroscience, 1, 216-219.

Naveh-Benjamin, M. (2000). Adult age differences in memory performance: Tests of an associative deficit hypothesis. Journal of Experimental Psychology: Learning, Memory, and Cognition, 26, 1170-1187.

Ohno, M. (2009). Failures to reconsolidate memory in a mouse model of Alzheimer's disease. Neurobiology of Learning and Memory, 92, 455-459.

Phelps, E. A., \& Hofmann, S. G. (2019). Memory editing from science fiction to clinical practice. Nature, 572, 43-50.

Rajah, M. N., Kromas, M., Han, J. E., \& Pruessner, J. C. (2010). Group differences in anterior hippocampal volume and in the retrieval of spatial and temporal context memory in healthy young versus older adults. Neuropsychologia, 48, 4020-4030.

Rindal, E. J., DeFranco, R. M., Rich, P. R., \& Zaragoza, M. S. (2016). Does reactivating a witnessed memory increase its susceptibility to impairment by subsequent misinformation? Journal of Experimental Psychology: Learning, Memory, and Cognition, 42, 1544-1558.

Sara, S. J. (2000). Retrieval and reconsolidation: toward a neurobiology of remembering. Learning \& Memory, 7, 73-84. 
Schacter, D. L., Guerin, S. A., \& St. Jacques, P. L. (2011). Memory distortion: An adaptive perspective. Trends in Cognitive Sciences, 15, 467-474.

Schwabe, L., \& Wolf, O. T. (2009). New episodic learning interferes with the reconsolidation of autobiographical memories. PLoS One, 4(10), e7519.

Sederberg, P. B., Gershman, S. J., Polyn, S. M., \& Norman, K. A. (2011). Human memory reconsolidation can be explained using the temporal context model. Psychonomic Bulletin \& Review, 18, 455-468.

St. Jacques, P. L., Montgomery, D., \& Schacter, D. L. (2015). Modifying memory for a museum tour in older adults: Reactivation-related updating that enhances and distorts memory is reduced in ageing. Memory, 23, 876-887.

St. Jacques, P. L., \& Schacter, D. L. (2013). Modifying memory: Selectively enhancing and updating personal memories for a museum tour by reactivating them. Psychological Science, 24, 537-543.

Wagenmakers, E.-J. et al. (2018). Bayesian inference for psychology. Part I: Theoretical advantages and practical ramifications. Psychonomic Bulletin \& Review, 25, 35-57.

Walker, M. P., Brakefield, T., Hobson, J. A., \& Stickgold, R. (2003). Dissociable stages of human memory consolidation and reconsolidation. Nature, 425, 616-620. 
Table 1. Means (and standard deviations) for age, MMSE, and NART for participants in Experiments 1 and 2.

\begin{tabular}{llll}
\hline & Age & MMSE & NART \\
\hline Young & $26.86(8.3)$ & N/A & N/A \\
Old & $73.43(8.13)$ & $29.4(1.2)$ & $43.15(5.19)$ \\
\hline
\end{tabular}


Table 2. Repeated Measures ANOVA for AB-AC learning trials, B and C recognition rates, and source monitoring as a function of Age, Reactivation, and Experiment.

\begin{tabular}{|c|c|c|c|c|c|c|c|c|c|c|c|c|c|c|}
\hline & & \multicolumn{6}{|c|}{ Experiment1 } & \multicolumn{7}{|c|}{ Experiment 2} \\
\hline & Age & & & Reac & & & Age* $^{*}$ & Age & & & Reac & & & Age* \\
\hline & Young & Old & $F$ & Reac & No Reac & $F$ & $F$ & Young & Old & $F$ & Reac & No Reac & $F$ & $F$ \\
\hline A-B Pairings & $2.81(1.18)$ & $4.06(1.28)$ & $47.09 * *$ & 3.4 (1.37) & $3.4(1.41)$ & 0.02 & 0.81 & $3.88(1.38)$ & $5.38(1.4)$ & $57.3 * *$ & $4.64(1.47)$ & $4.62(1.62)$ & 0.78 & 0.67 \\
\hline A-C Pairings & $2.84(0.98)$ & $4.23(1.27)$ & $75.9 * *$ & $3.45(0.97)$ & $3.5(0.92)$ & 0.71 & 0.11 & $3.91(1.31)$ & $5.32(1.32)$ & $57.2 * *$ & $4.6(0.92)$ & $4.57(1.28)$ & 0.36 & 0.63 \\
\hline Recognition B & $1(0)$ & $0.99(0.72)$ & 7.89 & $0.99(0.25)$ & $0.99(0.25)$ & 0.21 & 0.47 & $1(0)$ & $0.99(0.72)$ & 7.89 & $0.99(0.25)$ & $0.99(0.25)$ & 0.21 & 0.47 \\
\hline Recognition C & $1(0)$ & $0.98(0.37)$ & 1.54 & $0.99(0.78)$ & $0.99(0.71)$ & 0.32 & 0.44 & $1(0)$ & $0.98(0.25)$ & 1.54 & $0.99(0.78)$ & $0.99(0.71)$ & 0.32 & 0.44 \\
\hline Source B & $0.76(0.2)$ & $0.79(0.29)$ & 5.15 & $0.75(0.23)$ & $0.79(0.22)$ & 3.37 & 1.8 & $0.72(0.4)$ & $0.71(0.49)$ & 2.49 & $0.78(0.2)$ & $0.75(0.21)$ & 2.6 & 1.6 \\
\hline Source C & $0.71(0.24)$ & $0.69(0.22)$ & 1.99 & $0.68(0.27)$ & $0.67(0.29)$ & 0.48 & 2.2 & $0.64(0.24)$ & $0.66(0.42)$ & 4.23 & $0.67(0.42)$ & $0.66(0.64)$ & 1.23 & 0.76 \\
\hline
\end{tabular}


Figure 1. Materials for Experiment 1

\begin{tabular}{|c|}
\hline beaver \\
\hline boredom \\
\hline boy \\
\hline causality \\
\hline drama \\
\hline inn \\
\hline joviality \\
\hline lake \\
\hline market \\
\hline olive \\
\hline policeman \\
\hline shoes \\
\hline steamer \\
\hline strength \\
\hline sulphur \\
\hline tragedy \\
\hline
\end{tabular}
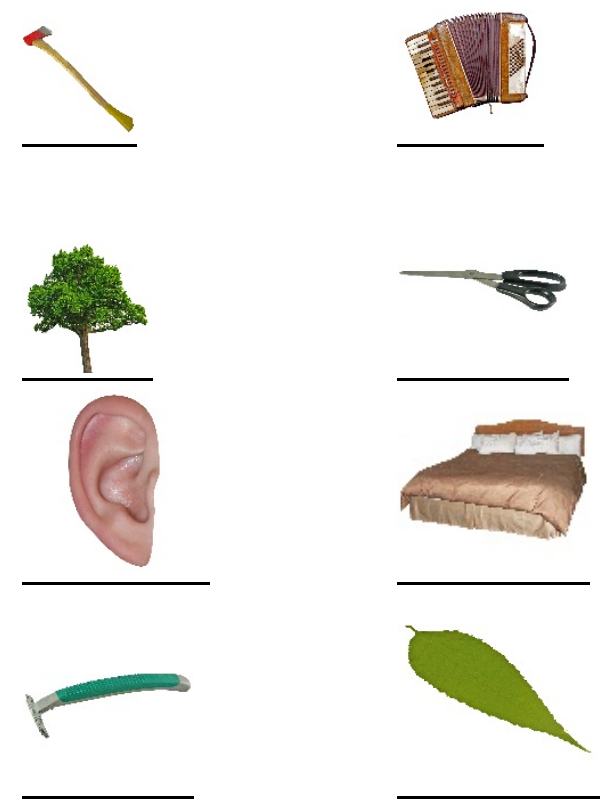
Figure 2. Learning Trials to Criterion at Session 2 as a Function of Age and Reactivation for the New C Terms in Experiment 1.

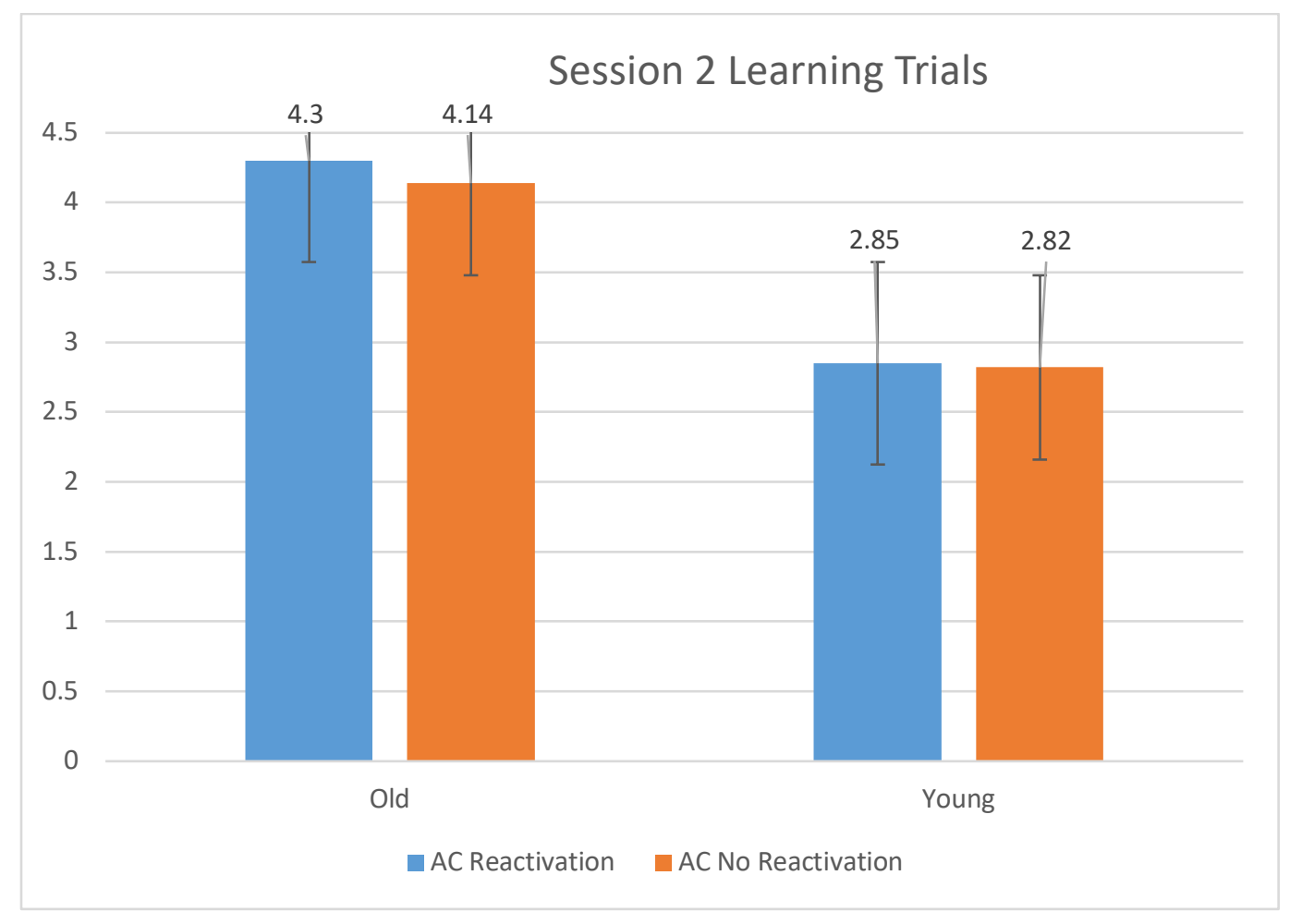


Figure 3. Materials for Experiment 2

\begin{tabular}{|c|}
\hline blap \\
\hline culp \\
\hline cusk \\
\hline derd \\
\hline dite \\
\hline dupe \\
\hline gake \\
\hline hage \\
\hline pisk \\
\hline posk \\
\hline$\underline{\text { reda }}$ \\
\hline roke \\
\hline sarn \\
\hline tand \\
\hline tust \\
\hline wupt \\
\hline
\end{tabular}

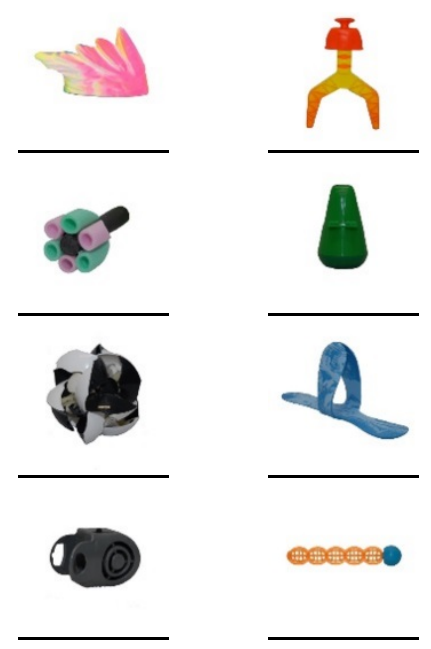


Figure 4. Learning Trials to Criterion at Session 2 as a Function of Age and Reactivation for the New C Terms in Experiment 2.

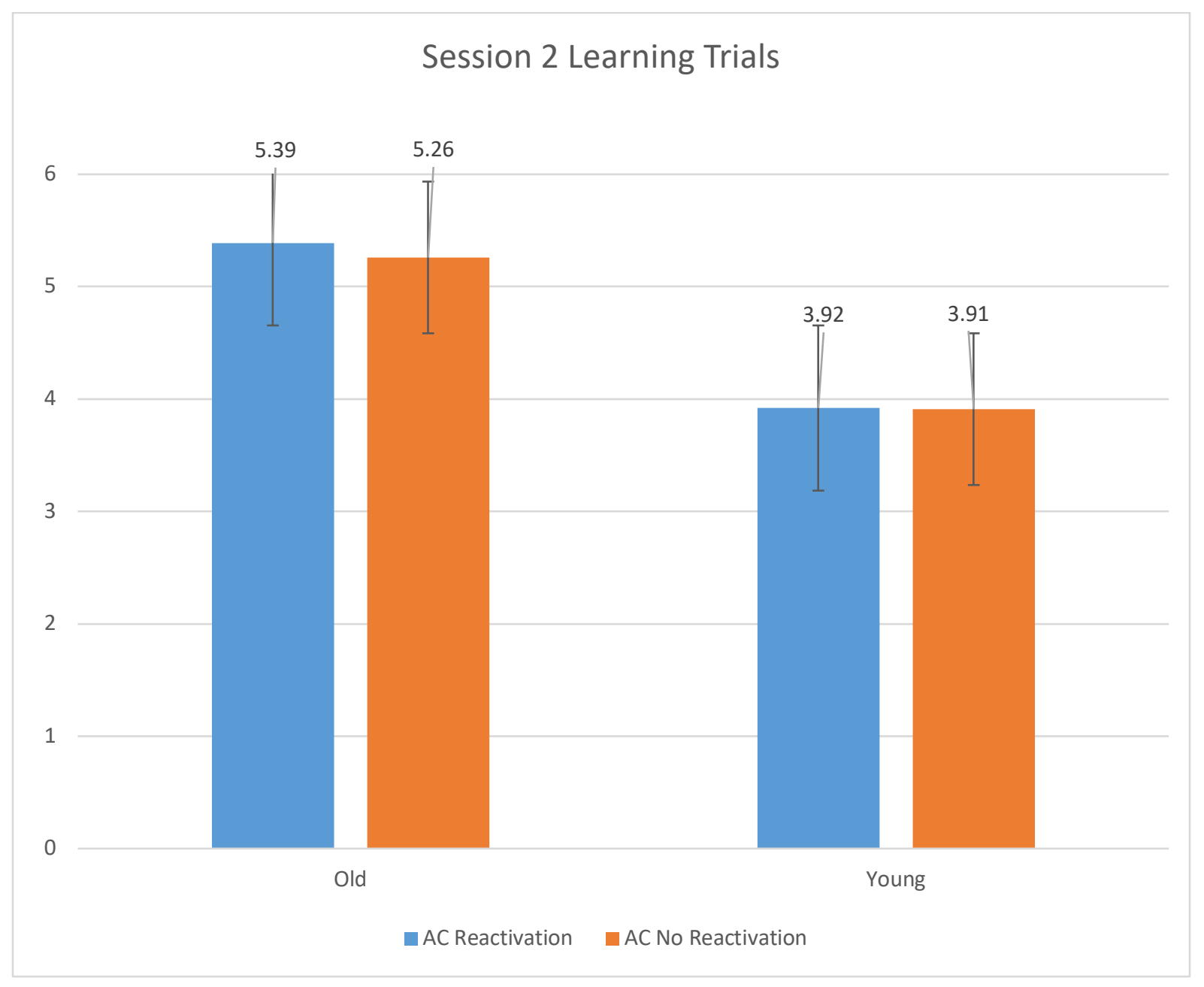




\section{Appendix}

\section{Exploratory Comparisons Between Experiments 1 and 2}

In order to examine whether there were differences in learning and recognition rates as a function of materials, we conducted repeated measures ANOVAs comparing Sessions 1, 2, and 3 across Experiments 1 and 2. For A-B pairings, there was a significant main effect for age $F(1,349)=103.1, p<.001, \eta_{p}^{2}=.19$ where younger adults had fewer learning trials. There was a main effect of experiment $F(1,349)=81.59, p<.001, \eta_{p}^{2}=.14$ where there were fewer learning trials in Experiment 1 than Experiment 2. There was no main effect of reactivation and no significant interactions (see Table 2 for all means).

For A-C pairings, there was a significant main effect for age $F(1,349)=130.42, p<$ $.001, \eta_{p}^{2}=.27$ where younger adults took fewer learning trials compared to the older adults. There was a main effect of experiment $F(1,349)=77.63, p<.001, \eta_{p}^{2}=.18$ where there were fewer learning trials in Experiment 1 than Experiment. There was no main effect of reactivation, and no significant interactions (see Table 2 for all means).

For the recognition test and source monitoring responses there was no significant main effect of experiment, reactivation, term, or age $p$ ns. There were no significant interactions (see Table 2 for all means). 